\title{
Atrial fibrillation prevalence, incidence and risk of stroke and all-cause death among Chinese ${ }^{\text {tr }}$
}

\author{
Kuo-Liong Chien ${ }^{\mathrm{a}, \mathrm{b}}$, Ta-Chen $\mathrm{Su}^{\mathrm{b}}$, Hsiu-Ching Hsu ${ }^{\mathrm{b}}$, Wei-Tien Chang ${ }^{\mathrm{c}}$, Pei-Chung Chen ${ }^{\mathrm{d}}$, \\ Ming-Fong Chen ${ }^{\mathrm{b}}$, Yuan-Teh Lee ${ }^{\mathrm{b}, *}$ \\ a The Institute of Preventive Medicine, College of Public Health, National Taiwan University, Taipei, Taiwan \\ ${ }^{\mathrm{b}}$ Department of Internal Medicine, National Taiwan University Hospital, Taiwan \\ ${ }^{\mathrm{c}}$ Department of Emergency Medicine, National Taiwan University Hospital, Taiwan \\ d Institute of Environmental Medicine, National Taiwan University, Taipei, Taiwan
}

Received 1 May 2008; received in revised form 15 July 2008; accepted 12 October 2008

\begin{abstract}
Background: We investigated atrial fibrillation (AF) prevalence, incidence and the risk of stroke and all-cause death because little is known about AF risk among ethnic Chinese.

Methods: We conducted a community-based prospective cohort study among 3560 participants. Prevalent and incident AF was documented by using the 12-lead ECG in baseline and serial follow-ups, and the stroke and all-cause death events were ascertained.

Results: Overall prevalence rates of $\mathrm{AF}$ in the cohort were $1.4 \%$ in men and $0.7 \%$ in women. Incidence rates of $\mathrm{AF}$ were 1.68 per 1000 person-years for men and 0.76 per 1000 person-years for women. During a median 13.8 years' follow-up, we documented 208 cases of stroke and 776 deaths. As compared with those without AF, participants with AF had nearly 4 times the age, gender-adjusted risk of stroke (relative risk [RR], 3.87, 95\% confidence interval [CI], 2.12-7.15), and twice the risk of death associated with all causes (RR, 2.23, 95\% CI, 1.523.27). Further adjustment for body mass index, lifestyle factors, socioeconomic status and clinical diseases slightly attenuated these risks. In addition, after adjusting for echocardiographic measures, the following risks remained significant: the multivariate RRs were 2.90 (95\% CI, 1.28-6.59) for risk of stroke and 2.05 (95\% CI, 1.27-3.32) for risk of all-cause death among participants with AF. Conclusion: Our data demonstrate that AF is a significant risk factor for stroke and all-cause death for the Chinese.

(C) 2008 Elsevier Ireland Ltd. All rights reserved.
\end{abstract}

Keywords: Atrial fibrillation; Community-based cohort; Chinese

\section{Introduction}

Atrial fibrillation (AF) is associated with congestive heart failure and thromboembolic mortality and morbidity, and is highly prevalent among the elderly population [1-3]. The substantial clinical impact and potential curable disease of

\footnotetext{
is This study was partially supported by the National Science Council (grant number: NSC 96-2314-B-002-155).

* Corresponding author. Department of Internal Medicine, National Taiwan University Hospital, Taipei, 100, Taiwan. Tel.: +886 22356 2910; fax: +886223920456

E-mail address: ytlee@ha.mc.ntu.edu.tw (Y.-T. Lee).
}

AF have made it mandatory for health professionals to understand the prevalence, incidence and associated factors of $\mathrm{AF}$ risk $[1,4]$. Although $\mathrm{AF}$ data on the Caucasian and African-American populations are available $[5,6]$, less is known about Asians and other ethnicities, especially from the community-based general population [7,8]. In addition, various risk factors associated with $\mathrm{AF}$ are also related to cardiovascular events and all-cause death in the general population and in specific clinical settings [9], such as older age, poor left ventricular function and hypertension $[2,10]$. Previous studies, based on cohort follow-ups in the general population or hospital setting $[11,12]$, have demonstrated 
that AF was a significant predictor for stroke and all-cause death. In addition, AF as a predictor for stroke and all-cause death in the Chinese general population is still unknown. Therefore, we investigated the AF risk based on the ChinShan Community Cardiovascular Cohort (CCCC) study, which provided a unique opportunity to explore the newonset AF using its serial follow-up and complete biochemical and anthropometric measurements of the adult participants living in the community.

\section{Methods}

\subsection{Study design and study participants}

Full details of the CCCC study can be found in other publications [13-16]. Briefly, the CCCC Study began in 1990 by recruiting 1703 men and 1899 women $\geq 35$ years old, homogeneous in Chinese ethnicity, and living in the Chin-Shan township $30 \mathrm{~km}$ north of metropolitan Taipei, Taiwan. Information about anthropometry, lifestyle, and medical conditions was assessed by interview questionnaires in two-year cycles, and the validity and reproducibility of the collected data and measurements have been reported in detail [16]. The National Taiwan University College of Public Health Committee Review Board approved the study protocol.

\subsection{Clinical measurements and echocardiographic measurements}

In the survey, all of the study participants were individually interviewed from a structured questionnaire. Information was collected on their socio-demographic characteristics, physical activity, smoking, alcohol drinking habits, dietary characteristics, personal and family histories of diseases, and hospitalizations. Their body mass index was recorded and calculated as weight (in kilograms)/height (in meters) in baseline and serial measurements. Blood pressure was taken after 10 min of rest and measured by a mercury sphygmomanometer with the subject seated comfortably, with arms supported and positioned at the level of the heart. Two blood pressure readings were taken in both arms. The cuff bladder was inflated quickly and then deflated $2 \mathrm{~mm} \mathrm{Hg}$ every second. If the readings varied by more than $10 \mathrm{~mm} \mathrm{Hg}$, an additional reading was taken. The disappearance of Korotkoff phase V sound was recorded as diastolic pressure. The medication rates of antihypertensive drugs were $11 \%$ in the study participants.

All echocardiographic studies were made with commercially available echocardiographs (HP2500) each equipped with a 2.5/2.0-MHZ phase-array transducer and a VHS videotape recorder as described in another study [17]. In brief, a two-dimensional parasternal long-axis view of left ventricle was obtained to adjust M-mode cursor position perpendicular to the interventricular septum and posterior wall of LV at the mitral valve chordal level. The left ventricular dimensions at end-diastole and at end-systole were measured and used to calculate left ventricular ejection fraction quantitatively. The ejection fraction was assessed by the area-length method in the apical four chamber view if regional wall motion abnormality was noted. Left ventricular mass was calculated from the Penn convention, according to the equation of Devereux and Reichek [18]. The intraclass correlation reliability was calculated by a simple replication one-way analysis of variance test. The values of correlation reliability were between 0.70 and $0.85[17]$.

\subsection{Identification of AF prevalence and incidence}

Participants were examined at baseline and in the biennial follow ups. Among the 3560 participants who had the available ECG data at baseline, 38 cases (1.07\%) showed AF. For serial ECG tracking, 3116 undertook the first followup ECG in 1992-93, 2988 participants had their second visit in 1994-1995, 2645 had their fifth ECG in 1999-2000. Hospital records were obtained for hospitalization, with

Table 1

Distribution of various baseline demographic, lifestyle, socioeconomic factors and clinical measures in the study population in the CCCC cohort (1990-91), specified by prevalent AF or not

\begin{tabular}{|c|c|c|c|}
\hline & \multirow{2}{*}{$\begin{array}{l}\text { No AF } \\
N=3522 \\
\end{array}$} & \multirow{2}{*}{$\begin{array}{l}\mathrm{AF} \\
N=38 \\
\end{array}$} & \multirow[t]{3}{*}{$P$} \\
\hline & & & \\
\hline & $\%$ & $\%$ & \\
\hline Gender & & & 0.047 \\
\hline Men & 47.0 & 63.2 & \\
\hline Women & 53.0 & 36.8 & \\
\hline Age (yr) & & & $<.0001$ \\
\hline $35-44$ & 25.3 & 2.6 & \\
\hline $45-54$ & 25.3 & 7.9 & \\
\hline $55-64$ & 26.1 & 26.3 & \\
\hline $65-74$ & 16.6 & 39.5 & \\
\hline$>=75$ & 6.7 & 23.7 & \\
\hline Currently smoking & 36.1 & 52.6 & 0.035 \\
\hline Alcohol intake & 29.7 & 39.5 & 0.19 \\
\hline Marital status & & & 0.16 \\
\hline Single & 2.9 & 2.6 & \\
\hline Lived with spouse & 85.9 & 76.3 & \\
\hline Divorced or separated & 11.2 & 21.1 & \\
\hline Education level & & & 0.40 \\
\hline$<9 \mathrm{yr}$ & 94.2 & 97.4 & \\
\hline$>=9 \mathrm{yr}$ & 5.9 & 2.6 & \\
\hline Job status & & & 0.012 \\
\hline No job & 49.7 & 73.7 & \\
\hline Blue collar & 34.5 & 15.8 & \\
\hline White collar & 15.8 & 10.5 & \\
\hline Regular exercise & 15.0 & 13.2 & 0.75 \\
\hline Hypertension & 29.8 & 52.6 & 0.002 \\
\hline Diabetes mellitus & 13.0 & 31.6 & 0.001 \\
\hline Poor LV ejection fraction $(<=40 \%)$ & 1.4 & 7.4 & 0.009 \\
\hline Electrographic LV hypertrophy & 7.9 & 10.5 & 0.55 \\
\hline History of stroke & 2.5 & 2.6 & 0.96 \\
\hline History of coronary heart disease & 4.7 & 10.5 & 0.10 \\
\hline
\end{tabular}

Abbreviation: LV, left ventricular. 
Table 2

Distribution of various baseline clinical and echocardiographic measures in the study population in the CCCC cohort (1990-91), specified by prevalent AF or not

\begin{tabular}{|c|c|c|c|c|c|c|c|}
\hline \multirow[t]{2}{*}{ Variable } & \multicolumn{3}{|c|}{ No $\mathrm{AF}$} & \multicolumn{3}{|l|}{$\mathrm{AF}$} & \multirow[t]{2}{*}{$P$} \\
\hline & $N$ & Mean & $\mathrm{SD}$ & $N$ & Mean & $\mathrm{SD}$ & \\
\hline Age, years & 3522 & 54.8 & 12.3 & 38 & 67.0 & 10.2 & $<.0001$ \\
\hline Body mass index, $\mathrm{kg} / \mathrm{m}^{2}$ & 3501 & 23.5 & 3.4 & 38 & 24.0 & 3.6 & 0.35 \\
\hline Systolic blood pressure, $\mathrm{mm} \mathrm{Hg}$ & 3505 & 125.5 & 20.5 & 38 & 138.3 & 28.6 & 0.009 \\
\hline Total cholesterol, mg/dL & 3500 & 198.1 & 45.4 & 38 & 172.7 & 31.8 & $<.0001$ \\
\hline Triglyceride, mg/dL & 3498 & 125.9 & 95.9 & 38 & 127.3 & 64.8 & 0.90 \\
\hline HDL-cholesterol, mg/dL & 3406 & 47.6 & 12.7 & 37 & 43.0 & 11.1 & 0.030 \\
\hline LDL-cholesterol, mg/dL & 3393 & 137.9 & 44.1 & 37 & 116.3 & 28.9 & $<.0001$ \\
\hline \multicolumn{8}{|l|}{ Echocardiographic measures } \\
\hline Left atrium, mm & 2652 & 33.0 & 5.7 & 27 & 45.5 & 10.1 & $<.0001$ \\
\hline IVS dimension, mm & 2622 & 10.4 & 2.4 & 27 & 11.6 & 3.2 & 0.06 \\
\hline LV posterior wall, $\mathrm{mm}$ & 2622 & 10.0 & 1.9 & 27 & 11.5 & 1.9 & $<.0001$ \\
\hline LVIDD, $\mathrm{mm}$ & 2622 & 46.0 & 5.6 & 27 & 48.7 & 6.5 & 0.012 \\
\hline LVIDS, mm & 2621 & 28.2 & 5.3 & 27 & 32.1 & 8.7 & 0.029 \\
\hline LV mass, $\mathrm{g}$ & 2622 & 193.9 & 67.5 & 27 & 259.6 & 106.3 & 0.004 \\
\hline End-diastolic volume, $\mathrm{mm}^{3}$ & 2622 & 99.3 & 27.9 & 27 & 114.0 & 35.2 & 0.007 \\
\hline End-systolic volume, $\mathrm{mm}^{3}$ & 2553 & 32.4 & 14.7 & 27 & 46.0 & 32.4 & 0.039 \\
\hline LV ejection fraction & 2621 & 0.68 & 0.10 & 27 & 0.62 & 0.16 & 0.05 \\
\hline
\end{tabular}

Abbreviation: SD, standard deviation; IVS, interventricular septum; LV, left ventricular; LVIDD, LV end-diastolic internal dimension; LVIDS, LV end-systolic internal dimension.

SI conversion factor: To convert triglycerides to mmol/L, multiply values by 0.01134 . To convert total, HDL, and LDL cholesterol to mmol/L, multiply values by 0.0259 .

adjudication of cardiovascular events by research committee [19]. Participants who were free from AF at baseline were defined as new-onset AF if AF was present on an electro- cardiogram obtained from the serial follow ups. The electrocardiographic interpretation of AF was confirmed by one of three cardiologists $(\mathrm{KC}, \mathrm{TS}$, or $\mathrm{WC})$.
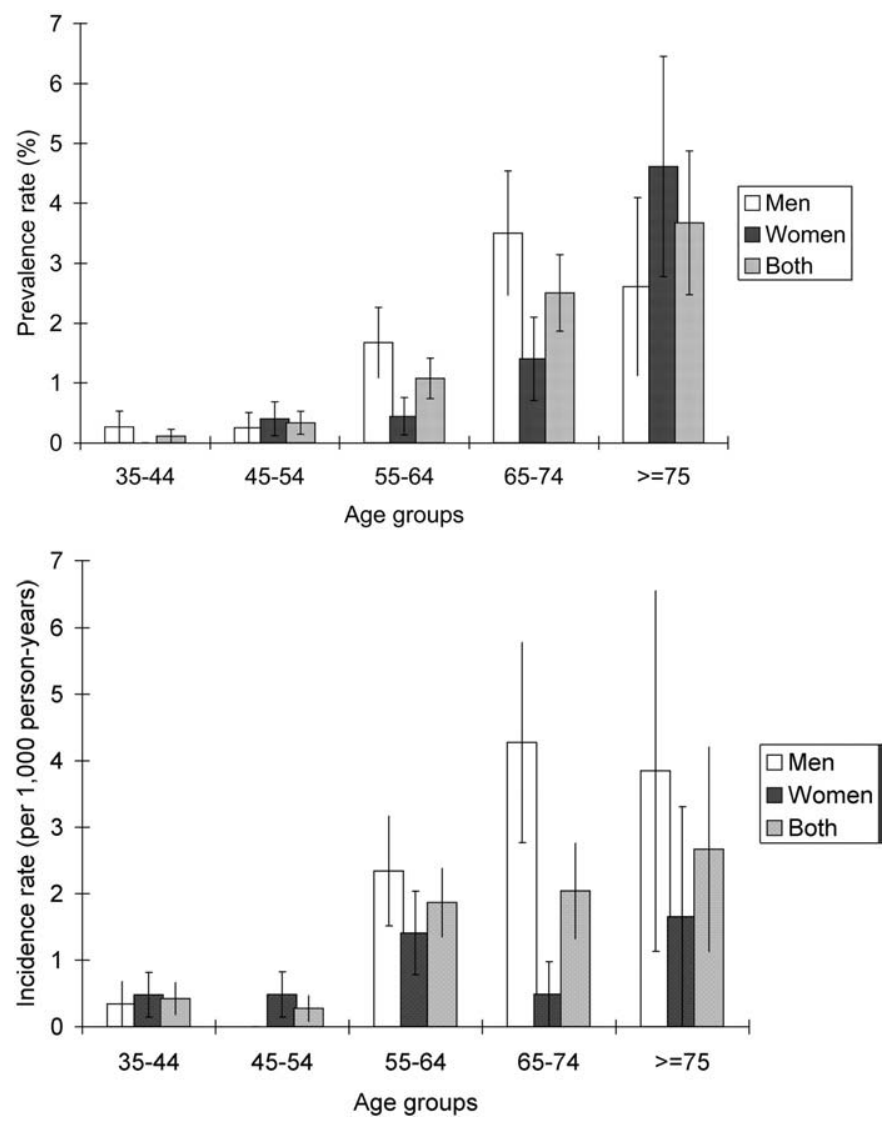

Fig. 1. Histograms of the prevalence rates in the baseline (upper, 1990-1991) and the incidence rates of atrial fibrillation during a median of 9.0 years' follow-up period (lower, 1990-2000) of the study population. The $95 \%$ confidence intervals were estimated according to the exact binomial test. 


\subsection{Outcome ascertainment and definition}

Incident CHD cases were defined by nonfatal myocardial infarction, fatal coronary heart disease and hospitalization due to percutaneous coronary intervention and coronary bypass surgery [13]. Fatal CHD was considered to have occurred if fatal myocardial infarction was confirmed by hospital records or if CHD was listed as the cause of death on the death certificate as the underlying and most plausible cause of death, or if evidence of previous CHD was available. Incident stroke cases were ascertained according to the following criteria: a sudden neurological symptom of vascular origin that lasted longer than $24 \mathrm{~h}$, with supporting evidence from the image study [19]. Transient ischemic attacks were not included in this study. The cases were confirmed by internists. Deaths were identified from official certificate documents, further verified by house-to-house visits.

\subsection{Statistical analysis}

The gender and age specific prevalence and incidence rates of $\mathrm{AF}$ were plotted and $95 \%$ confidence intervals were presented and estimated according to the exact binomial test. The distributions of demographics, lifestyle factors, socioeconomic status, clinical measures and echocardiographic measures were presented according to prevalent $\mathrm{AF}$ status. Incidence rates of stroke and all-cause death events were calculated as the number of events per 1000 person-years at risk. Kaplan-Meier estimated event-free survival functions were plotted and the log-rank test was applied. The Cox proportional-hazards regression model was used to estimate the relative risk (RR) and 95\% confidence interval (CI) associated with AF and the risk of stroke and all-cause death. We calculated the RR after adjusting confounding variables: Model 1 was adjusted for age groups (35-44, 45-54, 55-64, $65-74,>=75$ years old) and gender only. Model 2 included additional confounding factors: body mass index $(<18,18$ to $20.9,21$ to $22.9,23$ to 24.9 , or $>=25 \mathrm{~kg} / \mathrm{m}^{2}$ ), smoking (yes/ no or abstinence), current alcohol drinking (regular/no), marital status (single, married and living with spouse, or divorced and separated), education level (less than 9 years, at least 9 years), occupation (no work, labor, office, or business), regular exercise habit (yes/no). Model 3 included more clinical covariates such as baseline hypertension (yes/ no, defined by blood pressure at least 140/90 $\mathrm{mm} \mathrm{Hg}$ or on medication), diabetes mellitus (yes/no, defined by fasting plasma glucose at least $126 \mathrm{mg} / \mathrm{dL}$ or on medication), and history of stroke and coronary heart disease. Model 4 included additional electrocardiographic left ventricular hypertrophy, and echocardiographic measures as left atrium, left ventricular mass and left ventricular ejection fraction in the model among the participants with available echocardiographic measures. We developed the scoring system according to CHADS2 risk score[20], i.e., assigning 1 point each for the presence of congestive heart failure (defined as $\mathrm{LVEF}<40 \%$ ), hypertension, age 75 years or older, and diabetes mellitus and assigning 2 points for history of stroke or transient ischemic attack in applying to the subcohort who undertook echocardiographic data $(n=2648)$.

We used the area under the curve of receiver operator characteristics (ROC curve) to compare the discriminative ability of various risk factors [21]. The area under the ROC curve was considered as a global performance indicator for a prognostic factor [22]. We calculated several performance measures to check the predictive power of AF for outcomes, including a reclassification table [23], Hosmer-Lemeshow statistics, net reclassification improvement (NRI) and integrated discrimination improvement (IDI), which provided new insight on model comparison and prediction [24]. Briefly, the NRI was a sum of differences between the 'upward' movement in categories for event subjects and the "downward' movement in those for nonevent subjects [24]. We assumed the a priori risk categories according to quartiles and evaluated the NRI values in different risk settings. The IDI was viewed as a difference between improvement in average sensitivity and any potential

Table 3

Incident cases, person-years, rates, relative risks, and 95\% confidence intervals of stroke and all-cause death rates during a median of 13.8 years' period according to prevalent $\mathrm{AF}$ status in the CCCC study participants

\begin{tabular}{lllll}
\hline & $\mathrm{AF}(-)$ & $\mathrm{AF}(+)$ & & \\
\hline Stroke & & & & \\
Cases & 196 & 12 & & \\
Person-year & 44010.0 & 318.6 & & \\
Rates (/1000) & 4.5 & 37.7 & & \\
Relative risk & & & $95 \% \mathrm{CI}$ & $P$ \\
$\quad$ Model 1 & 1 & 3.87 & $(2.12-7.05)$ & $<.0001$ \\
$\quad$ Model 2 & 1 & 3.85 & $(2.11-7.05)$ & $<.0001$ \\
$\quad$ Model 3 & 1 & 3.58 & $(1.92-6.66)$ & $<.0001$ \\
$\quad$ Model 4 & 1 & 2.90 & $(1.28-6.59)$ & 0.011 \\
All-cause death & & & & \\
Cases & 748 & 28 & & \\
Person-year & 44661 & 340.0 & & \\
Rates (/1000) & 16.7 & 82.3 & & \\
Relative risk & & & $95 \% \mathrm{CI}$ & $P$ \\
$\quad$ Model 1 & 1 & 2.23 & $(1.52-3.27)$ & $<.0001$ \\
$\quad$ Model 2 & 1 & 2.03 & $(1.38-2.98)$ & 0.0003 \\
$\quad$ Model 3 & 1 & 1.96 & $(1.33-2.89)$ & 0.001 \\
$\quad$ Model 4 & 1 & 2.05 & $(1.27-3.32)$ & 0.003 \\
\hline
\end{tabular}

Model 1 was adjusted for age groups $(35-44,45-54,55-64,65-74$, $>=75$ years old) and gender only.

Model 2 included additional confounding factors: body mass index $(<18,18$ to $20.9,21$ to $22.9,23$ to 24.9 , or $>=25 \mathrm{~kg} / \mathrm{m}^{2}$ ), smoking (yes $/$ no or abstinence), current alcohol drinking (regular/no), marital status (single, married and living with spouse, or divorced and separated), education level (less than 9 years, at least 9 years), occupation (no work, labor, office, or business), and regular exercise habit (yes/no).

Model 3 included more clinical covariates as baseline hypertension(yes/no, defined by blood pressure at least $140 / 90 \mathrm{~mm} \mathrm{Hg}$ or on medication), diabetes mellitus (yes/no, defined by fasting plasma glucose at least $126 \mathrm{mg} / \mathrm{dL}$ or on medication), and history of stroke and coronary heart disease.

Model 4 included additional electrocardiographic left ventricular hypertrophy, and echocardiographic measures as left atrium, left ventricular mass and left ventricular ejection fraction in the model among the participants with available echocardiographic measures $(n=2679)$. 
increase in average 'one minus specificity' [24], and the statistic was a difference in Yates discrimination slopes between the new and old models $[25,26]$.

All statistical tests were two-tailed and probability values less than 0.05 were considered statistically significant. Analyses were performed with SAS version 9.1 (SAS Institute, Cary, NC) and Stata version 9.1 (Stata Corporation, College Station, Texas).

\section{Results}

Of the 3560 participants with available electrographic data at baseline, 38 had established AF rhythm. Table 1 shows the baseline characteristics of study participants specified by prevalent AF status. Participants with prevalent AF were more likely to be male, of older age, white collar or no job status, and smokers, and more likely to have hypertension, diabetes and poor left ventricular ejection fraction than those without prevalent AF. The lifestyle factors, including alcohol intake and exercise habits, and socioeconomic status, including marital status, education level, and history of stroke and coronary heart disease were similar between participants with and without AF. Participants with AF were more likely to have a high age systolic blood pressure, and lower cholesterol levels than those without AF (Table 2). With regards to echocardiographic measures, participants with AF had higher measures of left atrium, left ventricular septum, posterior wall, dimensions, and left ventricular mass, yet had a lower left ventricular ejection fraction than those without AF. Overall, the incidence rates of AF were 1.68 per 1000 person-years for men and 0.76 per 1000 person-years for women (Fig. 1). The incidence of AF increased appreciably with increasing age, and the highest incidence rates were 4.3 per 1000 personyears for men in the 65-74 year group and 1.7 per 1000 person-years for women in 75 years or older (Fig. 1, lower).

During a median 13.8 years' follow up (interquartile range, 13.5-14.6 years, total 45,001 person-years), we documented 208 cases of stroke, 122 cases of CHD and 776 deaths among the 3560 participants. The estimated cumulative event-free rate of stroke for participants with AF was $50 \%$, as compared with $90 \%$ for those without AF, and the
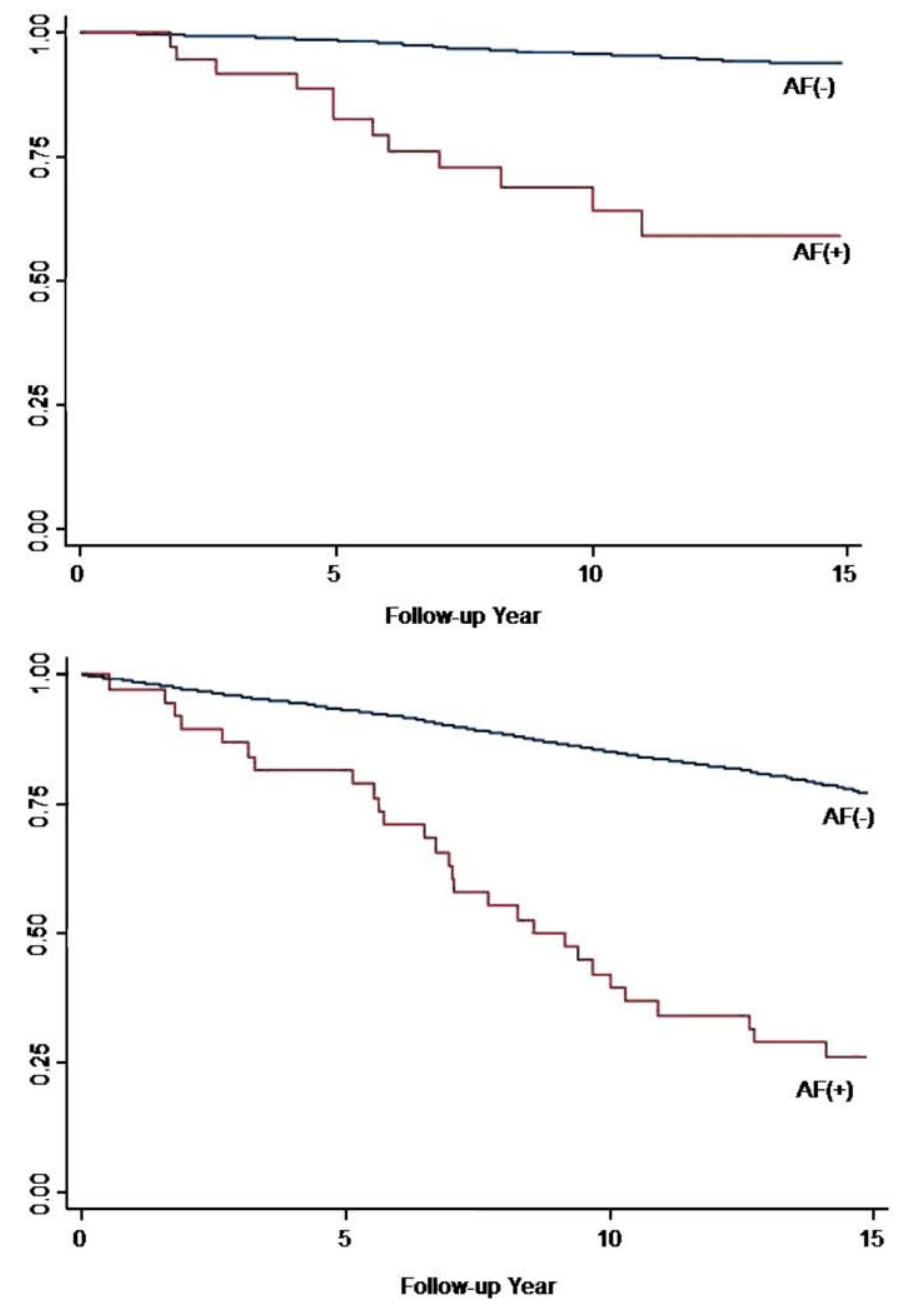

Fig. 2. The Kaplan-Meier estimated cumulative event-free rates with respect to stroke (upper) and all-cause death (lower) events in the study participants, according to prevalent AF status at baseline (both the log-rank test, $P<.0001$ ). 
cumulative event-free rate of all-cause death was similar (both the log-rank test, $P<0.0001$, Fig. 2). Incidence rates for stroke and all-cause death were significantly higher for participants with AF (Table 3): as compared with those without AF, participants with AF had nearly 4 times the age, gender-adjusted risk of stroke (RR, 3.87, 95\% CI, 2.127.15), and twice the risk of death associated with all causes (RR, 2.23, 95\% CI, 1.52-3.27). Further adjustments for body mass index, lifestyle factors, socioeconomic status and clinical diseases slightly attenuate the risks. In addition, after adjusting for echocardiographic measures including left atrium, left ventricular mass and ejection fraction status that are closely related with $\mathrm{AF}$, the following risks remained significant: the multivariate RRs were 2.90 (95\% CI, 1.28 6.59) for risk of stroke and $2.05(95 \% \mathrm{CI}, 1.27-3.32)$ for risk of all-cause death among participants with AF. However, we did not find significant association between AF and CHD risk (data not shown).

The mean (standard deviation) of CHADS2 score was $0.52(0.75)$, and the distribution range was $0-4$ in the study participants. Although the CHADS2 score was significantly associated with stroke risk (RR, 2.45, 95\% CI, 2.11-2.90, $P<.001)$ in the univariate model, the risk diminished appreciably after adjusting for other covariates. However, prevalent AF were still related to stroke risk in the same model (multivariate RR, 2.90, 95\% CI, 1.28-6.59, $P=.011$ ). We concluded that CHADS2 risk score didn't provide additional information for stroke prediction.

Table 4 shows the areas under the ROC curves, their Hosmer-Lemeshow goodness-of-fit statistics, integrated discrimination improvement, and net reclassification improvement between the extensive multivariate models with and without AF. The Hosmer-Lemeshow test did not reject the goodness of fit for any of the models. For stroke and all-cause death, the multivariate model with $\mathrm{AF}$

Table 4

Performance measures of multivariate models with and without AF for the risk of stroke and all-cause death among the study population

\begin{tabular}{llllllll}
\hline Outcome & Model & $\begin{array}{l}\text { Hosmer- } \\
\text { Lemeshow }\end{array}$ & $\begin{array}{l}\text { Area under } \\
\text { ROC curve }\end{array}$ & $\begin{array}{l}\text { IDI, } \\
\%\end{array}$ & $\begin{array}{l}\text { SE, } \\
\%\end{array}$ & $\begin{array}{l}\text { NRI, } \\
\%\end{array}$ & $\begin{array}{l}\text { SE, } \\
\%\end{array}$ \\
\hline Stroke & $\mathrm{AF}(+)$ & 0.752 & 0.814 & 0.83 & 0.75 & 0.94 & 1.70 \\
& $\mathrm{AF}(-)$ & 0.399 & 0.810 & & & & \\
Death & $\mathrm{AF}(+)$ & 0.406 & 0.841 & 0.55 & 1.09 & 0.47 & 0.80 \\
& $\mathrm{AF}(-)$ & 0.185 & 0.839 & & & & \\
\hline
\end{tabular}

Abbreviations: ROC, receiver operating characteristics; IDI, integrated discrimination improvement; NRI, net reclassification improvement; SE: standard error.

The multivariate model included: age groups (35-44, 45-54, 55-64, 65-74, $>=75$ years old $)$ gender, body mass index $(<18,18$ to $20.9,21$ to $22.9,23$ to 24.9 , or $>=25 \mathrm{~kg} / \mathrm{m}^{2}$ ), smoking (yes/no or abstinence), current alcohol drinking (regular/no), marital status (single, married and living with spouse, or divorced and separated), education level (less than 9 years, at least 9 years), occupation (no work, labor, office, or business), regular exercise habit (yes/no), clinical covariates as baseline hypertension (yes/no, defined by blood pressure at least $140 / 90 \mathrm{~mm} \mathrm{Hg}$ or on medication), diabetes mellitus (yes/no, defined by fasting plasma glucose at least $126 \mathrm{mg} / \mathrm{dL}$ or on medication), and history of stroke and coronary heart disease. outperformed the model without $\mathrm{AF}$ in predicting the outcomes - that is, the areas under their ROC curves are in each case larger than the areas under the ROC curves for their counterpart without AF. Although IDI and NRI values increased somewhat, they did not reach any significant difference (all $P>.05$ ). The NRI values for stroke and allcause death remained stable irrespective of risk category.

\section{Discussion}

In this prospective cohort of middle-aged to older ethnic Chinese, we clearly demonstrated AF as a significant predictor for stroke and all-cause death, after extensive adjustments. AF was also highly prevalent and incident among elderly Chinese adults.

Our findings are consistent with other population-based studies on the prevalence and incidence of AF risk [7,27]. With regards to the prevalence rates, our data were consistent with a cross-sectional survey data in the US [5] wherein the $\mathrm{AF}$ prevalence ranged widely from $0.1 \%$ among adults younger than 55 years to $9 \%$ among octogenarians. Age was an important determinant for AF risk. A survey on the Asian population reported relatively low rates for AF prevalence. A study on young healthy residents in northern India showed only $0.1 \%$ prevalence of AF [28]. Zhou and colleagues found that the age-standardized AF prevalence rate was only $0.61 \%$ among a representative Chinese sample of 2000 adults who were recruited randomly [29]. The Japanese survey showed a prevalence rate of $0.64 \%$ [30]. Our findings were also compatible with the European-based serial survey on participants of 50- to 89-year-olds, which showed AF incidence rates of $1.4-3.3 \%$ in men and $1.0-1.5 \%$ in women [31]. However, our findings showed an unexpectedly low incidence pattern of $\mathrm{AF}$ among ethnic Chinese. In the Framingham Heart Study that covered a 38-year follow-up period, Kannel and colleagues estimated the incidence of $2-$ 3 cases per 1000 person-years of AF among participants of 55-64 years old [2]. A European-based cohort composed of participants of 55 years and older showed an overall incidence AF rate of up to 9.9 per 1000 person-years. According to this European data, the lowest rate was 1.1 per 1000 person-years among 55-59 years old and their results were similar to our data in the corresponding age groups. Furthermore, Psaty and colleagues have demonstrated that African-American ethnicity was associated with a lower risk of AF than whites in an elderly population composed of 5201 participants older than 65 years [6]. The high incidence rates (12.0 per 1000 person-years among African-Americans and 19.5 per 1000 person-years) were explained by the advanced age in the study population. Another study among the longitudinal community-based older cohort of Olmsted County, Minnesota was composed of 4618 adults with an average of 73 years. The adjusted incidence rates were much lower, ranging from 3.04 to 3.68 per 1000 person-years [32], which was comparable to our data in a similar age group. The relatively lower $\mathrm{AF}$ incidence rate in our data may be 
attributed to the rather young age of the cohort participants and having only electrocardiographic evidence as outcome measurements. Another possible explanation for the low AF rate was the lower rate of congestive heart failure risk in our participants, compared with the Caucasian population [9].

Our findings support the notion that AF is a significant predictor for stroke and all-cause death. Ohsawa and colleagues collected single electrocardiographic AF data from a randomly selected sample of 9483 Japanese adults (older than 30 years) and examined the mortality causes nearly 20 years later [30]. AF was associated with stroke and all-cause mortality. Among 6417 older adult residents (mean 73 years old) who were diagnosed as having first-time atrial fibrillation in Olmsted County, Minnesota, and followed up for 5 years, an appreciably high risk of death developed in the early months, indicating a comorbidity status of new developed AF [33]. For the risk of stroke, AF was a predictive factor for stroke among 7547 Swedish middle-aged men during a 28-year follow-up period [34].

Our investigation cannot prove that an elevated body mass index is related to the prevalence and incidence of AF, although obesity was identified as an important risk factor [35-39]. The nonsignificant association might be attributable to the relatively low body mass index among our study participants, compared with Caucasians so that inconclusive result was found. We also demonstrated that blood pressure, hypertension, diabetes and left ventricular hypertrophy were associated with AF prevalence, consistent with other population-based studies $[5,8,40]$.

In our study, advanced age and male gender were significant risks for AF incidence, which was consistent with other studies. This is clearly an issue that deserves further attention as the elderly population continues to grow rapidly and public health burdens steadily increase. Regardless, elderly women also have high risks of developing AF and should be treated optimally for AF control $[41,42]$. The unmodifiable risk factors still have an important impact on AF incidence. Besides being male and having advanced age, lifestyle risk factors, including modest fish intake and moderate alcohol drinking, were associated with AF $[43,44]$. Our data showed that in addition to lifestyle factors and socioeconomic status, clinical hypertension, diabetes, and poor left ventricular ejection fraction were significantly associated with prevalent AF; these findings are consistent with previous studies [8,40]. Furthermore, various echocardiographic parameters, such as left atrium, left ventricular mass and ejection fraction, were appreciably associated with AF status [45]. Our data supported the additional information that echocardiography was a significant factor for further stroke and death events. Echocardiographic measurement for evaluating left ventricular function and structure could provide additional information for prognosis, which has become a gold standard for clinical practice.

To our knowledge, this is the first extensive investigation of the prevalence, incidence and effect on risk of stroke and allcause death for AF among ethnic Chinese. Because of the prospective cohort design, the baseline measurements of all cohort members were unlikely to be affected by exposure factors and laboratory issues. The use of a community-based population could reduce the possibility of selection bias. We also included important socioeconomic status, lifestyle factors, clinical measures and echocardiographic parameters in the models to control the potential confounding factors.

Our study had several potential limitations. First, the incident cases of AF events were relatively so small that the power to detect the risk of AF might have been reduced. Second, we did not specify AF cases into paroxysmal vs. chronic AF separately, similar to the limitation of the Framingham cohort [40]. Third, we did not investigate the anti-arrhythmic medication or other treatment modality histories among the study subjects. For newly diagnosed $\mathrm{AF}$, there exist efficient and useful treatment modalities for controlling the rhythm or rates [46]. We have no detailed treatment data for subjects who developed new-onset AF. Finally, with regards to the low antihypertensive medication rate in baseline, poor health service accessibility and low socioeconomic status of these participants may be the probable reasons. We didn't clarify the detailed list of medication.

In conclusion, our data provided prevalent $\mathrm{AF}$ data among ethnic Chinese and we have demonstrated that AF was appreciably associated with the risk of stroke and allcause death. Further research on AF prevention and treatment is warranted.

\section{Acknowledgements}

The authors thank the staff of the Department of Internal Medicine, National Taiwan University Hospital, and the participants of the CCCC study for their valuable contributions.

\section{References}

[1] Benjamin EJ, Levy D, Vaziri SM, et al. Independent risk factors for atrial fibrillation in a population-based cohort. The Framingham Heart Study. JAMA 1994;271:840-4.

[2] Kannel WB, Wolf PA, Benjamin EJ, et al. Prevalence, incidence, prognosis, and predisposing conditions for atrial fibrillation: population-based estimates. Am J Cardiol 1998;82:2N-9N.

[3] Stewart S, Hart CL, Hole DJ, et al. Population prevalence, incidence, and predictors of atrial fibrillation in the Renfrew/Paisley study. Heart 2001;86:516-21

[4] Iqbal MB, Taneja AK, Lip GY, et al. Recent developments in atrial fibrillation. BMJ 2005;330:238-43.

[5] Go AS, Hylek EM, Phillips KA, et al. Prevalence of diagnosed atrial fibrillation in adults: national implications for rhythm management and stroke prevention: the AnTicoagulation and Risk Factors in Atrial Fibrillation (ATRIA) Study. JAMA 2001;285:2370-5.

[6] Psaty BM, Manolio TA, Kuller LH, et al. Incidence of and risk factors for atrial fibrillation in older adults. Circulation 1997;96:2455-61.

[7] Wang TJ, Benjamin EJ. The epidemiology of atrial fibrillation. In: Kowey P, Naccarelli GV, editors. Atrial fibrillation. New York: Macel Dekker; 2005. p. 1-25.

[8] Chen LY, Shen WK. Epidemiology of atrial fibrillation: a current perspective. Heart Rhythm 2007;4:S1-6. 
[9] Huang CH, Chien KL, Chen WJ, et al. Impact of heart failure and left ventricular function on long-term survival-report of a communitybased cohort study in Taiwan. Eur J Heart Fail 2007;9:587-93.

[10] Tsang TS, Barnes ME, Gersh BJ, et al. Risks for atrial fibrillation and congestive heart failure in patients $>/=65$ years of age with abnormal left ventricular diastolic relaxation. Am J Cardiol 2004;93: $54-8$.

[11] Khairallah F, Ezzedine R, Ganz LI, et al. Epidemiology and determinants of outcome of admissions for atrial fibrillation in the United States from 1996 to 2001. Am J Cardiol 2004;94:500-4.

[12] Krahn AD, Manfreda J, Tate RB, et al. The natural history of atrial fibrillation: incidence, risk factors, and prognosis in the Manitoba Follow-Up Study. Am J Med 1995;98:476-84.

[13] Chien KL, Hsu HC, Su TC, et al. Lipoprotein(a) and Cardiovascular Disease in Ethnic Chinese: The Chin-Shan Community Cardiovascular Cohort Study. Clin Chem 2008;54:285-91.

[14] Chien KL, Hsu HC, Sung FC, et al. Incidence of hypertension and risk of cardiovascular events among ethnic Chinese: report from a communitybased cohort study in Taiwan. J Hypertens 2007;25:1355-61.

[15] Chien KL, Hsu HC, Su TC, et al. Apolipoprotein B and non-highdensity lipoprotein cholesterol and risk of coronary heart disease in Chinese. J Lipid Res 2007;48:2499-505.

[16] Lee YT, Lin RS, Sung FC, et al. Chin-Shan Community Cardiovascular Cohort in Taiwan: baseline data and five-year follow-up morbidity and mortality. J Clin Epidemiol 2000;53:836-46.

[17] Chien KL, Sung FC, Hsu HC, et al. Left Ventricular Mass and Correlated Atherosclerotic Risk Factors in Young Adolescents: Report from Chin-Shan Community Cardiovascular Study in Taiwan. Atherosclerosis 2001;155:431-7.

[18] Devereux RB, Reichek N. Echocardiographic determination of left ventricular mass in man anatomic validation of the method. Circulation 1977; 55:613-8

[19] Chien KL, Sung FC, Hsu HC, et al. Apolipoprotein A1 \& B, and stroke events in a community-based cohort in Taiwan: Report of Chin-Shan Community Cardiovascular Study. Stroke 2002;33:39-44.

[20] Gage BF, Waterman AD, Shannon W, et al. Validation of clinical classification schemes for predicting stroke: results from the National Registry of Atrial Fibrillation. JAMA 2001;285:2864-70.

[21] Hanley JA, McNeil BJ. A method of comparing the areas under receive operating characteristic curves derived from the same cases. Radiology 1983;148:839-43.

[22] Greiner M, Pfeiffer D, Smith RD. Principles and practical application of the receiver-operating characteristic analysis for diagnostic tests. Prev vet med 2000;45:23-41.

[23] Ridker PM, Buring JE, Rifai N, et al. Development and validation of improved algorithms for the assessment of global cardiovascular risk in women: the Reynolds Risk Score. Jama 2007;297:611-9.

[24] Pencina MJ, D' Agostino RBS, D' Agostino RBJ, et al. Evaluating the added predictive ability of a new marker: from area under the ROC curve to reclassification and beyond. Stat Med 2008;27:157-72.

[25] Yates J. External correspondence: decomposition of the mean probability score. Organ Behav Hum Perform 1982;30:132-56.

[26] Schmid C, Griffith J. Multivariable classification rules: calibration and discrimination. In: Armitage P, Colton T, editors. Encyclopedia of Biostatistics. Chichester, U.K.: Wiley; 1998.
[27] Kannel WB, Benjamin EJ. Status of the epidemiology of atrial fibrillation. Med Clin North Am 2008;92:17-40 ix.

[28] Kaushal SS, DasGupta DJ, Prashar BS, et al. Electrocardiographic manifestations of healthy residents of a tribal Himalayan village. J Assoc Phys India 1995;43:15-6.

[29] Zhou ZQ, Hu DY, Chen J, et al. [An epidemiological survey of atrial fibrillation in China]. Zhonghua Nei Ke Za Zhi 2004;43:491-4.

[30] Ohsawa M, Okayama A, Okamura T, et al. Mortality risk attributable to atrial fibrillation in middle-aged and elderly people in the Japanese general population: nineteen-year follow-up in NIPPON DATA80. Circ J 2007;71:814-9.

[31] Friberg J, Scharling H, Gadsboll N, et al. Sex-specific increase in the prevalence of atrial fibrillation (The Copenhagen City Heart Study). Am J Cardiol 2003;92:1419-23.

[32] Miyasaka Y, Barnes ME, Gersh BJ, et al. Secular trends in incidence of atrial fibrillation in Olmsted County, Minnesota, 1980 to 2000, and implications on the projections for future prevalence. Circulation 2006; 114:119-25.

[33] Miyasaka Y, Barnes ME, Bailey KR, et al. Mortality trends in patients diagnosed with first atrial fibrillation: a 21 -year community-based study. J Am Coll Cardiol 2007;49:986-92.

[34] Harmsen P, Lappas G, Rosengren A, et al. Long-term risk factors for stroke: twenty-eight years of follow-up of 7457 middle-aged men in Goteborg, Sweden. Stroke 2006;37:1663-7.

[35] Wanahita N, Messerli FH, Bangalore S, et al. Atrial fibrillation and obesity - results of a meta-analysis. Am Heart J 2008;155:310-5.

[36] Frost L, Hune LJ, Vestergaard P. Overweight and obesity as risk factors for atrial fibrillation or flutter: the Danish Diet, Cancer, and Health Study. Am J Med 2005;118:489-95.

[37] Wang TJ, Parise H, Levy D, et al. Obesity and the risk of new-onset atrial fibrillation. JAMA 2004;292:2471-7.

[38] Zacharias A, Schwann TA, Riordan CJ, et al. Obesity and risk of new-onset atrial fibrillation after cardiac surgery. Circulation 2005; 112:3247-55.

[39] Dublin S, French B, Glazer NL, et al. Risk of new-onset atrial fibrillation in relation to body mass index. Arch Intern Med 2006;166:2322-8.

[40] Mitchell GF, Vasan RS, Keyes MJ, et al. Pulse pressure and risk of new-onset atrial fibrillation. Jama 2007;297:709-15.

[41] Kerr CR, Humphries K. Gender-related differences in atrial fibrillation. J Am Coll Cardiol 2005;46:1307-8.

[42] Humphries KH, Kerr CR, Connolly SJ, et al. New-onset atrial fibrillation: sex differences in presentation, treatment, and outcome. Circulation 2001;103:2365-70.

[43] Mozaffarian D, Psaty BM, Rimm EB, et al. Fish intake and risk of incident atrial fibrillation. Circulation 2004;110:368-73.

[44] Mukamal KJ, Tolstrup JS, Friberg J, et al. Alcohol consumption and risk of atrial fibrillation in men and women: the Copenhagen City Heart Study. Circulation 2005;112:1736-42.

[45] Khumri TM, Idupulapati M, Rader VJ, et al. Clinical and echocardiographic markers of mortality risk in patients with atrial fibrillation. Am J Cardiol 2007;99:1733-6.

[46] Page RL. Clinical practice. Newly diagnosed atrial fibrillation. N Engl J Med 2004;351:2408-16. 